\title{
Identification of an ASC oligomerization inhibitor for the treatment of inflammatory diseases
}

\author{
Paula M. Soriano-Teruel ${ }^{1,2,7}$, Guillermo García-Laínez ${ }^{1,7}$, María Marco-Salvador ${ }^{1}$, Julián Pardo (iD) ${ }^{3}$, Maykel Arias ${ }^{3}$, Christian DeFord $^{4,5}$, \\ Irmgard Merfort ${ }^{4,5}$, María J. Vicent ${ }^{2}$, Pablo Pelegrín (D) ${ }^{6}$, Mónica Sancho (iD) ${ }^{{ }^{凶}}$ and Mar Orzáez (iD ${ }^{1 凶}$
}

(c) The Author(s) 2021

The ASC (apoptosis-associated speck-like protein containing a caspase recruitment domain (CARD)) protein is an scaffold component of different inflammasomes, intracellular multiprotein platforms of the innate immune system that are activated in response to pathogens or intracellular damage. The formation of ASC specks, initiated by different inflammasome receptors, promotes the recruitment and activation of procaspase-1, thereby triggering pyroptotic inflammatory cell death and proinflammatory cytokine release. Here we describe MM01 as the first-in-class small-molecule inhibitor of ASC that interferes with ASC speck formation. MM01 inhibition of ASC oligomerization prevents activation of procaspase-1 in vitro and inhibits the activation of different ASC-dependent inflammasomes in cell lines and primary cultures. Furthermore, MM01 inhibits inflammation in vivo in a mouse model of inflammasome-induced peritonitis. Overall, we highlight MM01 as a novel broad-spectrum inflammasome inhibitor for the potential treatment of multifactorial diseases involving the dysregulation of multiple inflammasomes.

Cell Death and Disease (2021)12:1155; https://doi.org/10.1038/s41419-021-04420-1

\section{INTRODUCTION}

ASC (apoptosis-associated speck-like protein containing a caspase recruitment domain (CARD)) is a protein of the innate immune system that participates in the formation of inflammasomes, which are macromolecular complexes responsible for the maturation and release of pro-inflammatory cytokines [1]. The inflammatory signaling engaged by inflammasome activation represents a rapid response that ensures the removal of detrimental stimuli and the repair of damaged tissue [2]. Therefore, inflammasome activation inhibits the spread of infection or the development of tumors and permits the development of an adaptive immune response [3]. However, inflammasome dysregulation has been implicated in the pathophysiology of conditions, such as Alzheimer's disease [4, 5], diabetes [6], and cancer [7, 8], and in the development of inflammatory/autoimmune disorders, such as gout, silicosis, rheumatoid arthritis, genetically inherited periodic fever syndromes [9-11], and severe acute respiratory syndromes in response to viral infections [12].

In detail, ASC is an adaptor protein that bridges different sensor receptors for pro-inflammatory stimuli, mainly the nucleotidebinding domain and leucine-rich repeat-containing receptor (NLR) sensor proteins, with the zymogen procaspase-1 (pro-Casp-1) [1]. NLRs oligomerize in response to specific conserved microbial structures known as pathogen-associated molecular patterns or danger-associated molecular patterns [13]. NLR oligomerization promotes ASC binding and oligomerization in large filaments that aggregate into large structures known as ASC specks, thereby generating a multitude of pro-Casp-1 interaction sites that promotes the auto-processing and activation of pro-Casp- 1 and, as a consequence, amplifying pro-inflammatory signaling [14, 15]. The ASC protein possesses two different protein domains, an $\mathrm{N}$-terminal pyrin (PYD) domain (ASC ${ }^{\mathrm{PYD}}$ ) and a C-terminal CARD domain (ASC ${ }^{C A R D}$ ). ASC ${ }^{\text {PYD }}$ nucleates filaments and ASC ${ }^{C A R D}$ links the oligomers to form specks [15-17]. Moreover, ASC CARD interacts with pro-Casp-1 ${ }^{\text {CARD }}$ to promote pro-Casp-1 oligomerization and activation and the subsequent processing and secretion of the mature forms of pro-inflammatory cytokines, such as interleukin (IL)-1 $\beta$ and IL-18 [18]. Of note, caspase-1 also cleaves Gasdermin D, which forms pores in the plasma membrane to trigger pyroptotic cell death [19-21]. ASC specks also possess an extracellular function that contributes to the spread of inflammatory signaling. ASC specks appear in the serum of cryopyrin-associated periodic syndrome patients [22] and in the cerebrospinal fluid of ischemic stroke model animal, where concentrations correlate with damage severity [23].

Several distinct inflammasome types have been described to date, with each differing with regards to the structure of the NLR component. The NLR family pyrin domain containing 1 (NLRP1), NLRP3, NLR family CARD domain-containing protein 4 (NLRC4), and interferon-inducible protein AIM2 inflammasomes are the most studied. The ASC dependence varies for each inflammasome,

\footnotetext{
${ }^{1}$ Targeted Therapies on Cancer and Inflammation Laboratory, Centro de Investigación Príncipe Felipe, Valencia, Spain. ${ }^{2}$ Polymer Therapeutics Lab., Centro de Investigación Príncipe Felipe, Valencia, Spain. ${ }^{3}$ Centro de Investigaciones Biomédicas de Aragón (CIBA), Universidad de Zaragoza, Zaragoza, Spain. ${ }^{4}$ Department of Pharmaceutical Biology and Biotechnology, Albert-Ludwigs-Universität, Freiburg, Germany. ${ }^{5}$ Spemann Graduate School of Biology and Medicine (SGBM), Albert-Ludwigs-Universität, Freiburg, Germany.

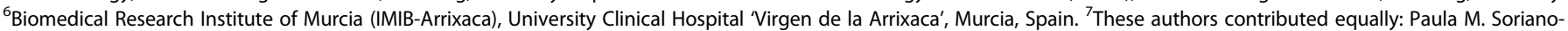
Teruel, Guillermo García-Laínez. ${ }^{凶}$ email: msancho@cipf.es; morzaez@cipf.es Edited by Professor Boris Zhivotovsky
} 
A

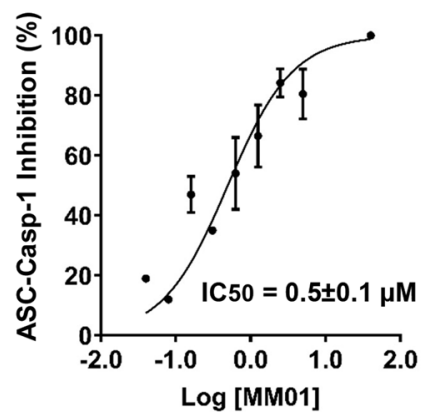

B

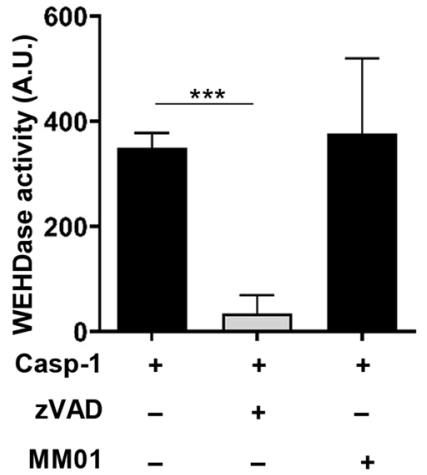

C

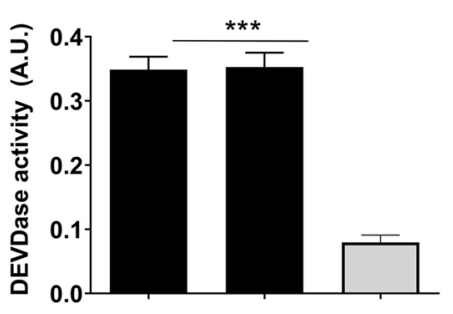

E
D

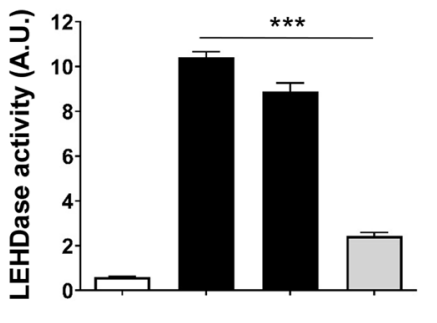

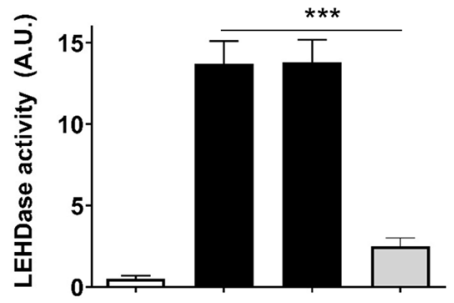

Procasp-9 + $+\quad+\quad+$

Apaf-1 - $+\quad+\quad+$

MM01 - $-\quad+\quad-$

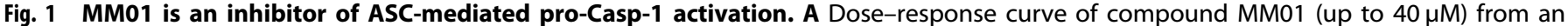

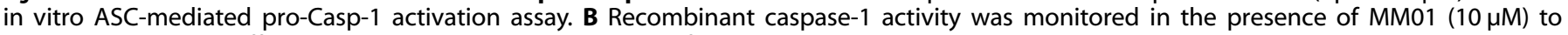

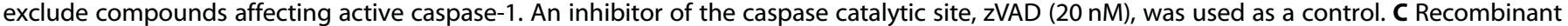

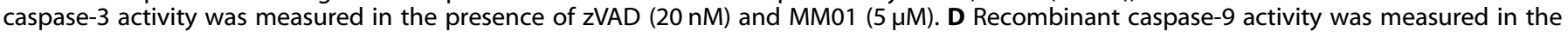

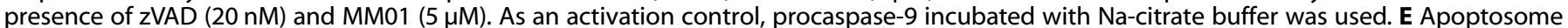

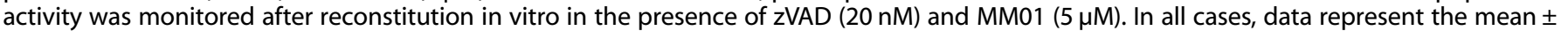
$\mathrm{SD}$ of three independent experiments $\left({ }^{* * *} p<0.001\right)$.

while pro-Casp-1 activation in NLRP3 and AIM2 inflammasomes requires ASC, the presence of ASC enhances pro-inflammatory signaling in CARD containing sensors, such as NLRP1 and NLRC4 $[14,15]$. Recently, structural studies showed direct interaction between NLRP1 ${ }^{\text {CARD }}$ and ASC CARD and between NLRC4 ${ }^{\text {CARD }}$ and $\operatorname{ASC}^{C A R D}[16,24]$. As inflammatory responses depend on the activation of more than one inflammasome in several diseases [25-27], any drug that can inhibit numerous inflammasome complexes simultaneously would be of significant interest.

Currently reported inflammasome inhibitors target the oligomerization of NLR (inflammasome-specific inhibitors) [28], caspase-1 [29], and downstream events in the inflammasome activation cascade, such as $\mathrm{IL}-1 \beta$ or its receptor [30], that compromise signaling events such as pyroptosis, which itself is responsible for the spread and amplification of inflammatory signaling. In this study, we identify MM01 as a modulator of inflammasome activity with a novel mechanism of action-the inhibition of ASC oligomerization and subsequent pro-Casp-1 activation. We demonstrate that MM01 disrupts ASC oligomerization triggered by different inflammasomes and inhibits downstream IL-1 $\beta / \mathrm{IL}-18$ release and pyroptosis in various cellular models of inflammation. Moreover, MM01 decreases neutrophil infiltration and pro-inflammatory cytokine accumulation in an in vivo model of peritonitis used as a proof of concept for the potential therapeutic capabilities of this novel inhibitor. Given the requirement for ASC in multiple inflammasome complexes, MM01 treatment may represent an effective treatment in a range of multifactorial diseases.

\section{RESULTS}

MM01 blocks ASC-mediated pro-Casp-1 activation in vitro To identify inhibitors of ASC oligomerization, we first screened for molecules that inhibited the activation of pro-Casp-1 by ASC in an in vitro assay using recombinant human ASC purified from Escherichia coli and pro-Casp-1 purified from Baculovirus (Fig. S1A). Details and development of the assay are summarized in Fig. S1A, $B$ and in the "Materials and methods" section. Once ASCdependent caspase-1 activation was established, we evaluated small-molecule compounds from the MyriaScreen Diversity Library I in dual-mixes and those active were deconvoluted to identify hit compounds that inhibited pro-Casp-1 by at least $60 \%$ (Fig. S1C, D). Positive hits were evaluated in a secondary screening against preactivated caspase- 1 to discard drugs targeting the active site of caspase-1 (Fig. S1E). To establish the best hit compound, we performed a drug-likeness score measured using the OSIRIS Property Explorer [31] (Fig. S1F). As a result of these assays, we identified MM01, a molecule that inhibited ASC-mediated proCasp- 1 activation exhibiting an $\mathrm{IC}_{50}$ in the nanomolar range (Fig. $1 \mathrm{~A}$ and Fig. S1G). We corroborated that MM01 do not targeted the active site of caspase-1, as opposed to the known caspase inhibitor zVAD (Fig. 1B). We also confirmed MM01 specificity via 
A

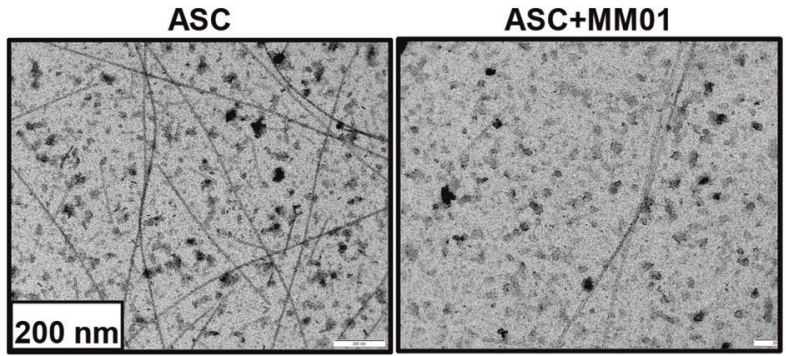

B $\mathrm{ASC}-++++$

MM01 - - + + -

Bafilomycin - $-\quad++$

ASC-YFP $\longrightarrow-55$
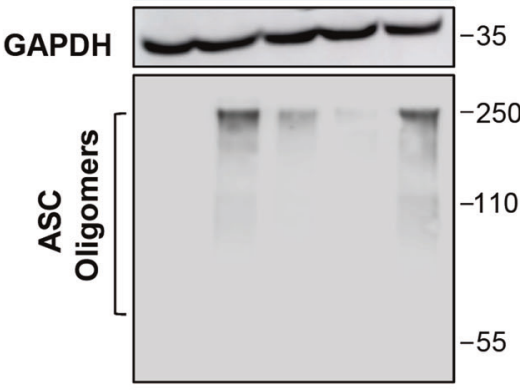

C

UNTREATED
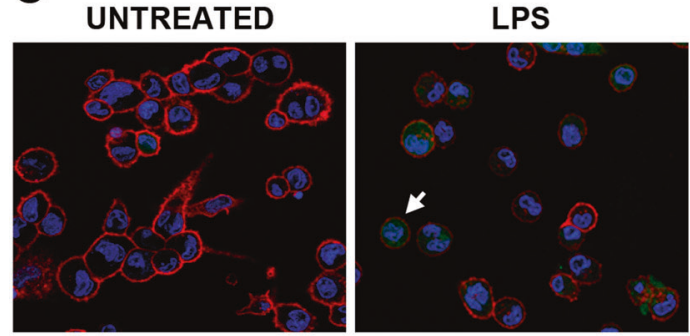

D

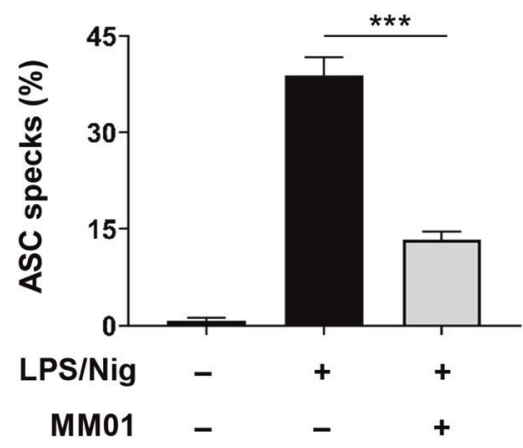

LPS/Nigericin

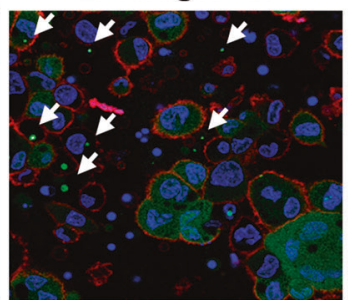

LPS/Nigericin+MM01

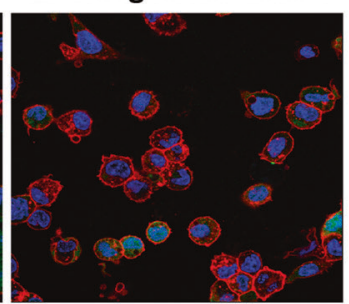

E

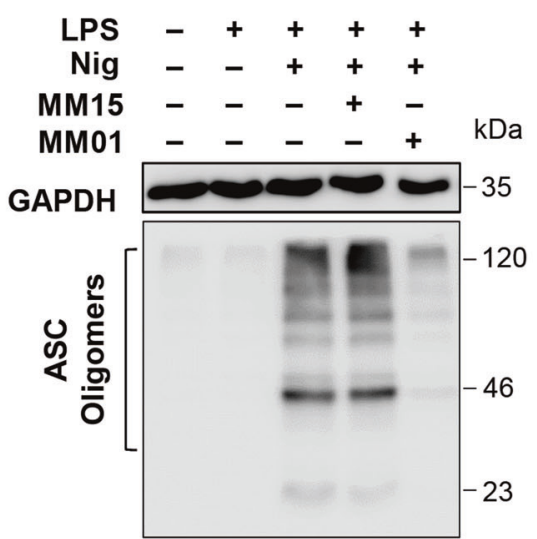

Fig. 2 MM01 inhibits ASC oligomerization in vitro. A Electronic microscopic images of negatively stained preparations of human recombinant ASC filaments in the presence of MM01 $(20 \mu \mathrm{M})$. B Western blot of lysates and crosslinked cytosolic pellets of ASC-YFP transfected HEK293 cells treated with MM01 $(10 \mu \mathrm{M})$. GAPDH was used as a loading control for lysate samples. C Live-cell imaging of THP-1 ASC GFP cells treated with MM01 $(10 \mu \mathrm{M})$ and stimulated with LPS $(100 \mathrm{ng} / \mathrm{ml})$ and nigericin $(10 \mu \mathrm{M})$. The blue signal corresponds to DAPI staining and the red signal to the cytoplasmic marker WGA (wheat germ agglutinin). D Percentage of ASC specks in the THP-1 ASC GFP cells treated as in C and measured by flow cytometry. Data represent the mean \pm SD of three independent experiments $(* * * p<0.001)$. E Western blot of crosslinked cytosolic pellets from PMA-differentiated THP-1 ASC GFP cells stimulated with LPS and nigericin and treated with the indicated compounds as in the above-described conditions.

the absence of inhibition of other apoptotic caspases, such as caspase-3 or caspase-9 (Fig. 1C, D). To confirm the non-specific inhibitory activity of MM01, we also analyzed apoptosome inhibition. The apoptosome shares structural characteristics with the inflammasome and acts as a molecular platform for caspase- 9 activation through CARD-CARD interactions. However, we did not observe any apoptosome inhibition in the presence of MM01 (Fig. $1 \mathrm{E})$, confirming a specific mechanism of action for MM01 on the ASC-mediated pro-Casp-1 activation.

\section{MM01 prevents ASC oligomerization}

We anticipated two possible mechanisms of action for MM01: the inhibition of ASC-pro-Casp-1 interactions or the interference of ASC oligomer formation that would lead to impaired recruitment and activation of pro-Casp-1. To explore the ability of MM01 to interfere in ASC oligomerization and speck assembly, we reconstituted ASC filaments in vitro from purified human recombinant ASC protein either in the presence or absence of MM01. Analysis by electron microscopy showed that the assembly of ASC specks was severely affected by the presence of MM01, thereby confirming the interference with ASC speck formation (Fig. 2A).

To corroborate the mechanism of action in the cellular milieu we transiently transfected ASC-YFP into HEK293 cells. HEK293 cells lack all the components of the inflammasome, and ASC-YFP transfection leads to spontaneous speck formation [32]. Interestingly in these assays, MM01 treatment avoided the formation of ASC oligomers (Fig. 2B); however, we observed that treatment with MM01 produced a decrease in ASC protein levels. The 
existence of a transcriptional regulatory effect of the MM01 compound was promptly discarded as no changes were observed in ASC mRNA levels, measured by quantitative reverse transcription polymerase chain reaction (Fig. S2A, B). Therefore, we hypothesized that MM01 could modify ASC to prevent oligomer formation and induce protein degradation. To confirm this hypothesis, and given the involvement of the lysosomal pathway in ASC degradation [33], we treated ASC-YFP-transfected HEK293 cells with bafilomycin, an inhibitor of autophagosome-lysosome fusion, alongside MM01 treatment. Bafilomycin treatment induced the recovery of ASC protein levels without the restoration of ASColigomerization following MM01 treatment, thereby confirming that MM01 interferes with ASC speck formation and facilitates ASC degradation in HEK293 cells (Fig. 2B).

Next, we studied the ability of MM01 to inhibit ASC speck formation using THP1-ASC-GFP cells, a cell line derived from THP-1 human monocytic cells that stably express an ASC-GFP fusion protein under the control of nuclear factor (NF)-KB-binding promoter. In resting cells, we did not observe any fusion protein; however, a priming signal (lipopolysaccharide (LPS) treatment) induces NF-KB-dependent ASC-GFP expression, while activation of the NLRP3 inflammasome by nigericin stimulation causes ASC-GFP speck formation. We monitored the ASC-GFP oligomerization upon treatment with both LPS and nigericin by confocal microscopy (white arrows in Fig. 2C) and observed a reduction of ASC speck formation following MM01 treatment (Fig. S2C). Quantification of ASC speck content by flow cytometry confirmed a $>50 \%$ reduction of specks in MM01-treated cells (Fig. 2D).

To corroborate this observation, we chemically crosslinked THP1 cell pellets and isolated ASC oligomers [34]. LPS and nigericin strongly induced ASC oligomerization, which was not altered following the treatment of cells with a negative control compound from the screening (MM15) (Fig. 2E). Indeed, only MM01 treatment strongly inhibited ASC oligomerization (Fig. 2E).

Docking studies with GOLD 5.2 (Protein Ligand Docking Software [35]) located critical interactions between ASC and MM01 involving residues His-118, Trp-169, Lys-174, Leu-177, Leu178, and Leu-192 (Fig. 3A), which correspond to ASC CARD domain. Mutations of residues Trp-169 or Arg-119 (near His-118) prompt the disruption of oligomerization [16]. Therefore, drugs such as MM01 may target these residues to impair oligomer formation and interfere with pro-Casp-1 activation.

To investigate the relevance of these residues in ASC speck formation, we mutated these positions by site-directed mutagenesis in the ASC-YFP construct to study ASC oligomer formation in HEK293. Site-directed mutagenesis rendered the $A C^{\mathrm{H} 118 \mathrm{~A}}$,

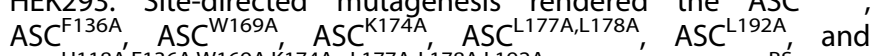

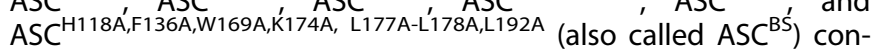
structs. Additionally, mutated residue R119 was used as control because of its described inability to form specks [16]. HEK293 cells were transfected with these mutants and speck formation was analyzed by flow cytometry. Interestingly, with the exception of the more conservative mutations $A S C\left[{ }^{L 177 A, L 178 A}\right]$, all other mutations produced a significant reduction in the number of ASC specks (Fig. 3C, D) without affecting protein expression (Fig. 3B), thereby indicating that the MM01 binding pocket is critical for ASC speck formation and represents a new site for therapeutic intervention.

\section{MM01 inhibits ASC-mediated inflammatory signaling}

After demonstrating the molecular mechanism of action for MM01, we evaluated inflammasome inhibitory activity in THP-1 cells differentiated with phorbol 12-myristate 13-acetate (PMA) as a model of human macrophage function.

THP-1-derived-macrophages primed with LPS and activated by the specific NLRP3 activation stimulus nigericin exhibited an MM01-dependent reduction of IL-1 $\beta$ and IL-18 secretion (Fig. 4A and Fig. S3E). Interestingly, MM01 significantly reduced the levels of pyroptosis as measured by decreased lactate dehydrogenase $(\mathrm{LDH})$ release (Fig. 4B). As pyroptosis contributes to the amplification of the pro-inflammatory signaling to neighboring cells [36], the MM01-mediated inhibition of pyroptosis could significantly dampen inflammatory spread. As expected, treatment with MM01 reduced the presence of active caspase- 1 and processed IL-1 $\beta$ in cell supernatants without affecting the expression of other inflammasome components (Fig. 4C). We also confirmed the ability of MM01 to inhibit the NLRP3 inflammasome using another specific NLRP3-activating stimulus, such as ATP [37] (Fig. S3A-D). Thus, MM01, which interferes with ASC speck formation, inhibits NLRP3-dependent inflammasome activity.

Next, we evaluated the ability of MM01 to inhibit the activity of other ASC-dependent and ASC-enhanced inflammasome complexes. Treatment of THP-1 cells with LPS and muramyl dipeptide (MDP) activates the NLRP1 inflammasome [38]; under these conditions, MM01 pretreatment avoided pro-Casp-1 activation, IL$1 \beta$ and IL-18 release, and protected cells from pyroptotic cell death (Fig. 4D, G and Fig. S4A, B). Cytosolic double-stranded DNA triggers the AIM2 inflammasome in PMA-differentiated THP-1 cells and induces IL-1 $\beta$ release. Due to the ASC dependence of this inflammasome, we also found that MM01 treatment significantly decreased IL-1 $\beta$ secretion (Fig. $4 \mathrm{E}, \mathrm{H}$ ). In the same line of evidence, MM01 treatment impaired IL-1 $\beta$ maturation and release following Salmonella typhimurium-induced NLRC4 activity [39] in THP-1 cells, leading to decreased pyroptosis (Fig. 4F, I and Fig. S4C).

Overall, these results confirm MM01 as an inhibitor of ASC oligomerization with the ability to inhibit the activity of a broad spectrum of inflammasomes.

MM01 inhibits inflammasome activation in primary cultures After demonstrating the activity of MM01 in established cell lines, we moved to evaluate MM01 in more physiological models of inflammation. We first evaluated the activity of MM01 in human peripheral blood mononuclear cells (PBMCs) stimulated with LPS and nigericin or ATP to activate the NLRP3 inflammasome. Encouragingly, treatment of these primary cultures with MM01 produced a significant decrease in IL-1 $\beta$ release and pyroptosis (Fig. 4J, K and Fig. S5A, B). ASC speck formation in this model is also compromised by the presence of the compound (Fig. $4 \mathrm{~L}$ ), strengthening our results.

As we aimed to study the activity of MM01 in animal models of inflammation, we next confirmed the response of murine cells to MM01 treatment. For this purpose, we evaluated NLRP3 inhibition in murine peritoneal macrophages stimulated with LPS and ATP, with MM01 treatment significantly inhibiting both IL-1 $\beta$ levels and subsequent IL-6 release [40] (Fig. 5A, B). Importantly, we did not find a significant reduction in tumor necrosis factor-alpha (TNF-a) release (Fig. 5C), indicating the specific activity of MM01 as an inhibitor of inflammasome signaling. We also found a significant decrease in $\mathrm{LDH}$ release in MM01-treated murine macrophages (Fig. 5D), thereby confirming the inhibition of pyroptosis by MM01 in murine immune cells.

\section{MM01 inhibits NLRP3 activation in vivo}

Finally, we evaluated the efficacy of MM01 in a murine monosodium urate (MSU)-induced model of peritonitis [41]. The particulate structure of MSU crystals produces a very potent activation of NLRP3 [42]. In this experimental model, the intraperitoneal injection of MSU crystals induces peritoneal inflammation, which can be followed by the increase in IL-1 $\beta$ levels and neutrophil infiltration in mice intraperitoneal fluid. The number of infiltrating neutrophils has been correlated with the extent of inflammasome activation [43]. Interestingly, treatment with MM01 $(10 \mathrm{mg} / \mathrm{kg})$ efficiently suppressed MSU-induced IL-1 $\beta$ production and peritoneal neutrophil recruitment (Fig. 5E, F), with no effect on IL-6 and TNF-a secretion (Fig. S5C, D), thereby 
A
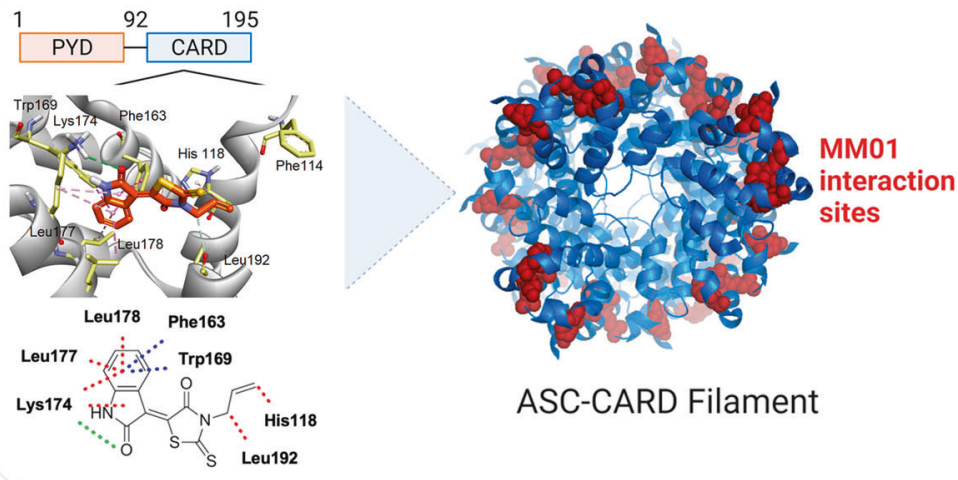

ASC-CARD Filament

B
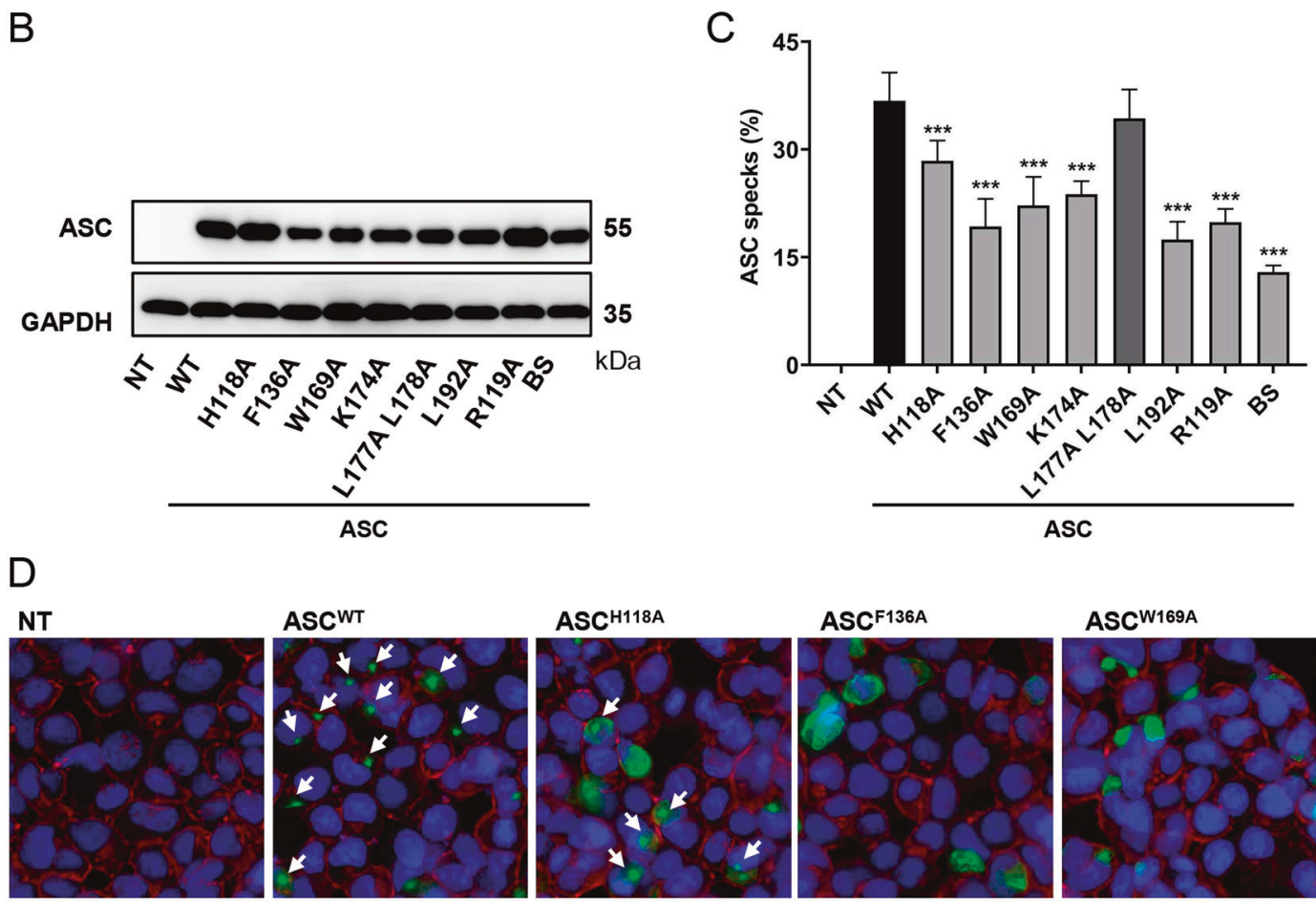

ASCW169A
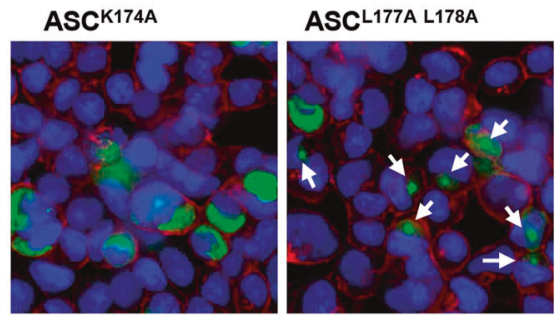

\section{ASCL192A}

ASCR119A
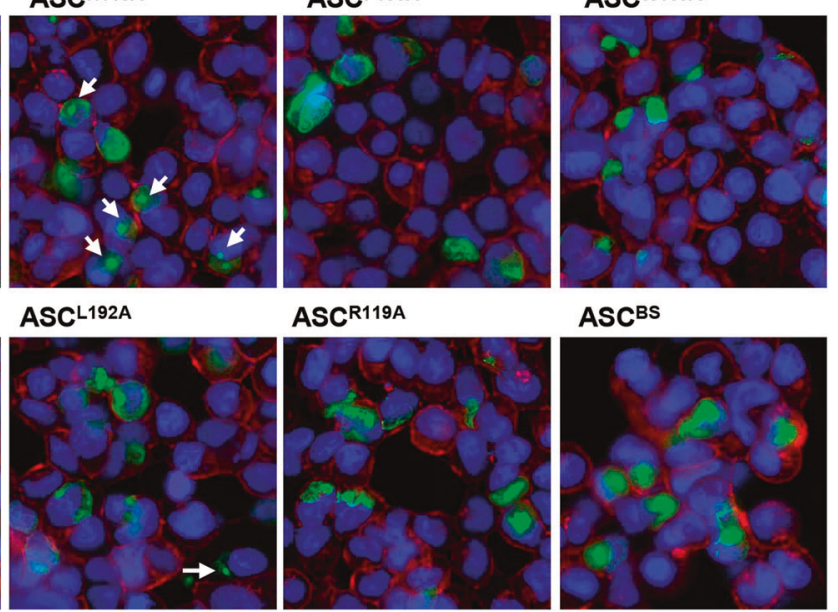

ASCBS

Fig. 3 ASC-MM01-binding site characterization. A Docking model of ASC with MM01. Green lines represent hydrogen bonds, red lines show van der Waals interactions, and blue lines depict $\pi$ interactions. Western blot (B) and percentage (C) of ASC specks in HEK293 cells transfected with the different ASC mutants and measured by flow cytometry. Data represent the mean \pm SD of three independent experiments $\left(^{* * *} p<\right.$ 0.001). D Live-cell imaging of HEK293 cells transfected with ASC mutants. The blue signal corresponds to DAPI staining and the red signal to the cytoplasmic marker WGA.

providing evidence for the in vivo anti-inflammatory activity of the ASC speck inhibitor MM01.

\section{DISCUSSION}

In MM01, we describe a novel ASC inhibitor that avoids ASC speck formation and impedes pro-Casp-1 activation. Modulation of the inflammasome at the ASC level causes efficient inhibition of caspase- 1 and cytokine release and significantly reduces pyroptotic cell death. Of note, MM01 treatment can inhibit inflammation both in vitro and in vivo in a mouse model.

While drugs that target IL-1 $\beta$ signaling, such as antibodies (canakinumab) or recombinant IL-1 $\beta$ receptor antagonists (anakinra), have found use as effective treatments for human inflammatory diseases, they do not cover all consequences of inflammasome activation, such as the additional proinflammatory cytokine release, the extracellular accumulation of ASC specks, or pyroptotic cell death, which all contribute to the 
A

B

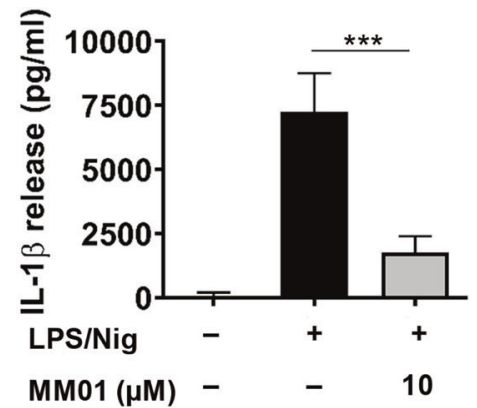

D

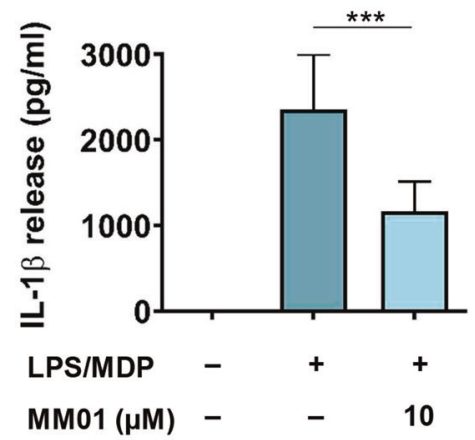

G

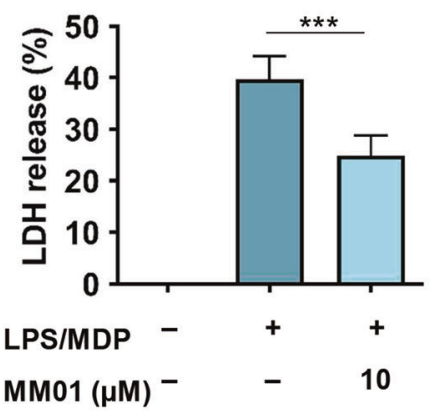

$J$

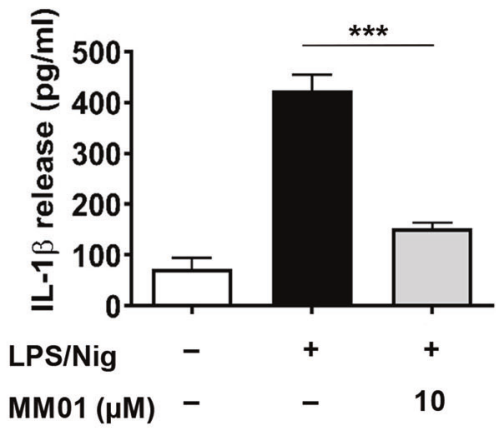

$\mathrm{H}$

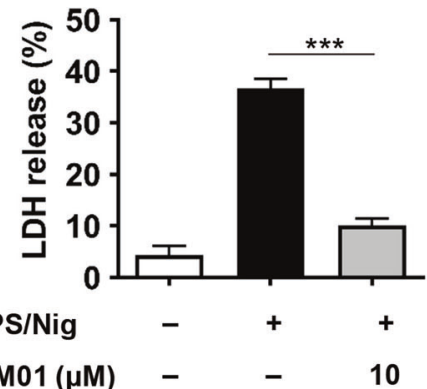

$\mathrm{E}$

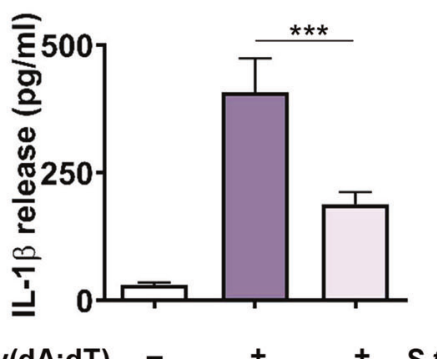

C

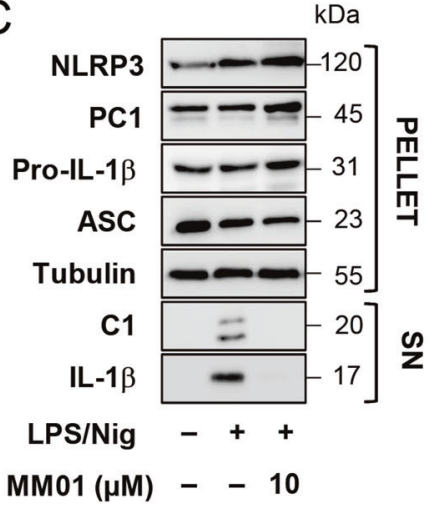

$\mathrm{F}$
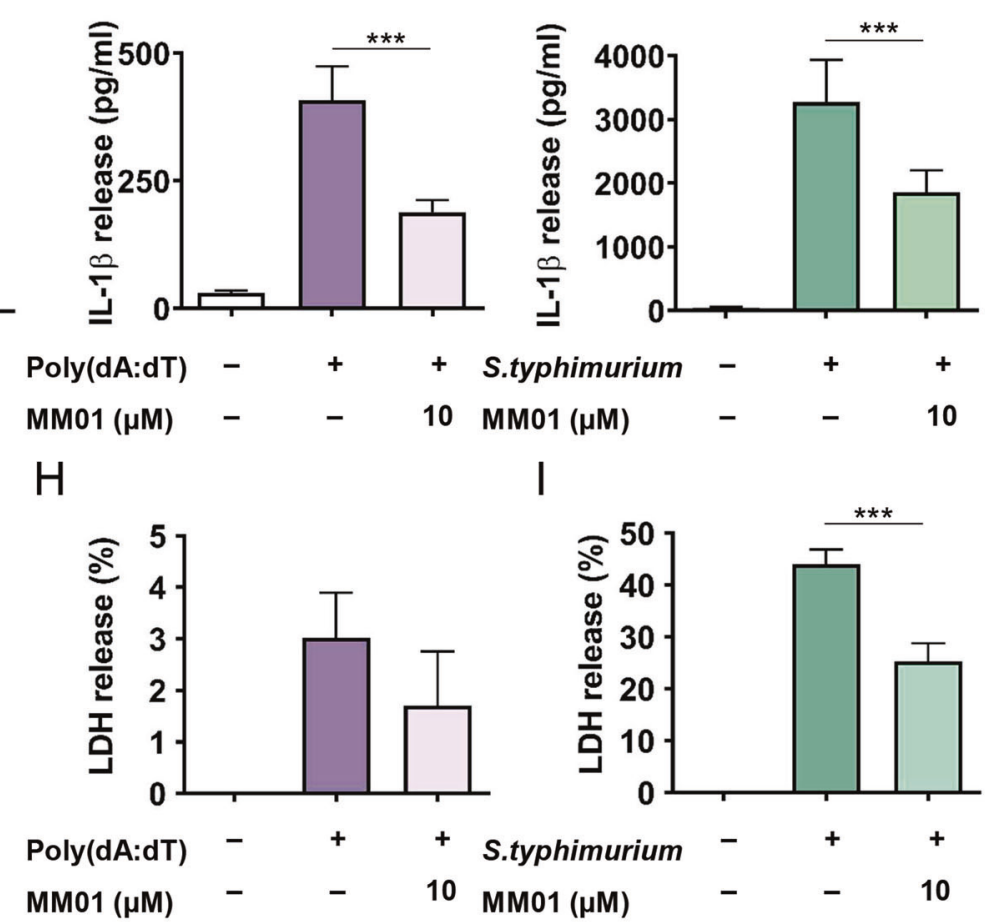

I

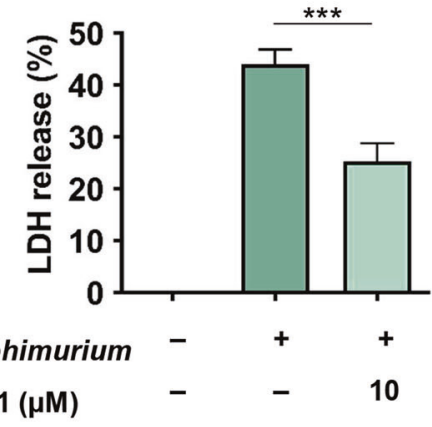

$\mathrm{L}$

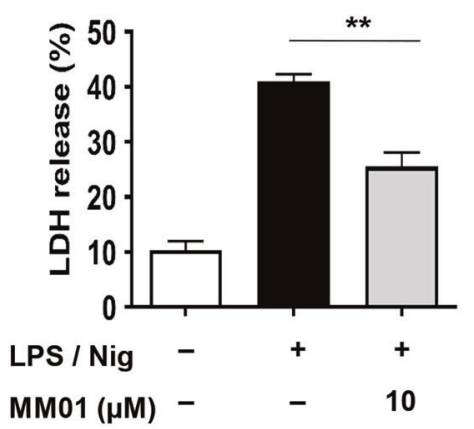

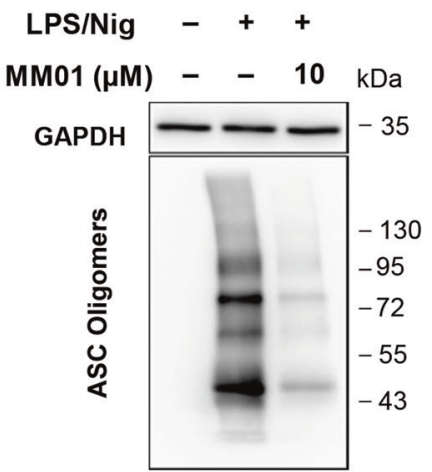

spread of the pro-inflammatory signaling [44]. Our results reinforce the hypothesis that the perturbation of ASC interaction interfaces could represent a target for the development of broadspectrum anti-inflammatory agents with improved cell recovery capabilities.
This inhibitor is predicted to act on a novel site on the ASC surface that impedes oligomerization, avoiding pro-Casp-1 recruitment and activation. The CARD domain of ASC is capable of forming filaments [16]. Amino acids involved in this interaction are close to those involved in the MM01 binding site. In particular, the 
Fig. 4 MM01 inhibits ASC-dependent inflammasome activity in vitro. A IL-1 $\beta$ secretion was evaluated by ELISA following activation of the NLRP3 inflammasome with LPS $(100 \mathrm{ng} / \mathrm{ml})$ and nigericin (Nig; $10 \mu \mathrm{M})$. Cells were treated with MM01 at $10 \mu \mathrm{M}$. B Measurement of LDH release into the extracellular medium under the above-described conditions. C THP-1 cells were stimulated as described above, and supernatants (SN) and pellets were analyzed by immunoblotting for IL-1 $\beta$ and cleaved caspase-1. A representative blot is shown. IL-1 $\beta$ measured by ELISA (D) and LDH release (G) were evaluated upon activation of the NLRP1 inflammasome with LPS (100 $\mathrm{ng} / \mathrm{ml})$ and MDP $(50 \mu \mathrm{g} / \mathrm{ml})$ in THP-1 cells. Cells were treated with MM01 at $10 \mu \mathrm{M}$. IL-1 $\beta$ secretion $(\mathbf{E})$ and LDH release $(\mathbf{H})$ were analyzed upon stimulation of the AIM2 inflammasome with poly (dA:dT) $(200 \mu \mathrm{g} / \mathrm{ml})$ in PMA-differentiated THP-1 cells treated or not with MM01 at $10 \mu \mathrm{M}$. IL-1 $\beta$ (F) and LDH secretion (I) were evaluated upon NLRC4 activation mediated by Salmonella typhimurium in THP-1 cells. Cells were treated with MM01 at $10 \mu \mathrm{M}$. MM01 inhibits LPS/Nig stimulation of isolated human PBMCs. IL-1 $\beta(\mathbf{J})$ and LDH (K) release were evaluated upon activation of the NLRP3 inflammasome with LPS $(100 \mathrm{ng} / \mathrm{ml})$ and nigericin $(10 \mu \mathrm{M})$ and $+/-$ MM01 at $10 \mu \mathrm{M}$ in human PBMCs. L Western blot of crosslinked cytosolic pellets from PBMCs stimulated as described above. In all cases, asterisks represent significant differences to the stimulated control as determined by a one-way ANOVA with Tukey's multiple comparisons test ${ }^{* *} p<0.05 ;{ }^{* *} p<0.001$. All data expressed as mean \pm SD of three independent experiments.

W169 residue is involved in type II (hydrophobic) interactions that maintain the structure of said filament. R119, close to Histidine 118 , is involved in type I interactions (charge-charge interactions) that stabilize the interaction between the helices of two ASC molecules. Further structural studies are needed to better understand how the named residues participate in oligomerization or structure stabilization. Here we have demonstrated that modulation at this level causes efficient inhibition of caspase-1 and cytokine release. Then, the results presented in this study validate the molecular target ASC and the drug, MM01, as a hit for further development into a lead compound. Of course, the activity of MM01 is limited to ASC-dependent inflammasomes, and in consequence some diseases where non-ASC dependent inflammasomes are involved will require the development of other specific drugs.

In line with our data, a related study has described the development of a single domain antibody fragment that specifically recognizes the CARD domain of human ASC [45]. This antibody impairs ASC (CARD) interactions, inhibits inflammasome activation, and protects cells from inflammatory cell death. Additionally, ASC inhibition by treatment with an IC-100 antiASC monoclonal antibody has prompted clear improvements in mice models of multiple sclerosis [46]. Recent research has also begun to describe ASC functions independent of the inflammasome. For example, the presence of ASC-deficient CD8+ T cells in transplanted mice decreases rejection responses, thereby suggesting a role for ASC in the modulation of cytotoxic T lymphocyte activity [47]. Thus, molecules targeting ASC such as MM01 could represent a new approach to inhibit transplant rejection. Interestingly, Schneider et al. recently established that ASC specks could activate Casp-8 and induce secondary pyroptosis in the absence of Casp-1 [36]. The inhibition of ASC by MM01 may effectively block secondary pyroptosis in this case.

Furthermore, growing evidence has implicated the involvement of numerous inflammasome types in human diseases. The activity of multiple inflammasomes has been implicated in inflammatory bowel disease in the regulation of commensal microbiota [48-50], while NLRP3, NLRP6, and NLRC4 expression can influence tumor formation [10]. Furthermore, the activity of at least two inflammasome types contribute to type 2 diabetes and other metabolic disorders $[6,51]$. Both NLRP3 and NLRC4 have been described roles in neuroinflammation, with double knockouts shown to prompt improvements in astrogliosis and microglial accumulation [26]. While inflammasome inhibitors such as MCC950 [52] or CY-09 [53] have specific and highly effective anti-inflammatory effects on the activation of the NLRP3 inflammasome, conditions that involve more than one inflammasome type require a more general inhibitory strategy.

In light of the current circumstances, we note the involvement of multiple inflammasomes in viral infections [54]. Studies of severe acute respiratory syndrome coronavirus- 2 indicate that virus severity correlates with an exacerbated inflammatory response, in which ASC specks play a fatal role [55]. Preclinical investigations with MM01 may contribute to a deeper understanding of viral infection mechanisms and offer novel effective therapeutic alternatives.

\section{MATERIALS AND METHODS}

\section{Purification of procaspase-1 (pro-Casp-1)}

Human His-tagged pro-Casp-1 was previously cloned into the pFastBac vector for Baculovirus expression. The expression plasmid was transformed into $\mathrm{DH} 10 \mathrm{Bac} E$. coli cells. Recombinant bacmids were then purified and used to transfect Sf9 insect cells. The virus stock was amplified and used to infect suspension cultures. Briefly, Sf9 cells were grown in $2 \mathrm{~L}$ of Grace's Insect Medium (Gibco) and infected at 1,500,000 cells $/ \mathrm{ml}$ with a recombinant virus that contained His-tagged pro-Casp-1 at a multiplicity of infection (MOI) of 1 . Cells were then cultured for $16 \mathrm{~h}$ at $27^{\circ} \mathrm{C}$ with constant gentle agitation to induce protein expression. Next, cells were centrifuged at $1000 \times g$ for $10 \mathrm{~min}$ at $4{ }^{\circ} \mathrm{C}$ and washed with phosphatebuffered saline (PBS). The pellet was lysed with buffer lysis (100 mM HEPES$\mathrm{KOH} \mathrm{pH} \mathrm{7.5,50} \mathrm{mM} \mathrm{KCl,} 7.5 \mathrm{mM} \mathrm{MgCl}, 5 \mathrm{mM} \mathrm{NaEDTA}, 5 \mathrm{mM} \mathrm{NaEGTA}$, and protease inhibitors: Pepstatin, Leupeptin, and PMSF) using a Douncer and clarified by centrifugation $\left(10,000 \times g, 1 \mathrm{~h}\right.$, at $\left.4^{\circ} \mathrm{C}\right)$. The resulting supernatant that contained the recombinant protein was purified in a Ni-NTA (Ni2p-nitrilotriacetate)-agarose column. After elution, pro-Casp-1 was further purified by ion-exchange chromatography (Mono Q) in $20 \mathrm{mM}$ of HEPES-KOH pH 7.5, $10 \mathrm{mM} \mathrm{KCl}, 1.5 \mathrm{mM} \mathrm{MgCl}, 1 \mathrm{mM}$ EDTA, and 1 mM EGTA.

\section{Purification of ASC}

ASC was previously cloned into the pET28a vector for expression in E. coli. Briefly, $8 \mathrm{~L}$ of $\mathrm{LB}$ medium, plus the antibiotics chloramphenicol and kanamycin, were inoculated with an overnight culture of freshly transformed His tagged-ASC-pET28a-BL21 (DE3) pLys E. coli (Invitrogen) and grown at $37^{\circ} \mathrm{C}$ until optical density (OD) 0.7. ASC expression was induced with $0.7 \mathrm{mM}$ isopropyl $\beta$-D-1-thiogalactopyranoside (IPTG) at $28^{\circ} \mathrm{C}$ for $4 \mathrm{~h}$. Cells were harvested $\left(15 \mathrm{~min}, 6000 \mathrm{rpm}\right.$ at $\left.4^{\circ} \mathrm{C}\right)$, lysed in $300 \mathrm{ml}$ of Buffer A (50 mM NaH$\left.{ }_{2} \mathrm{PO}_{4}, 300 \mathrm{mM} \mathrm{NaCl}, \mathrm{pH} 8.0\right)$, and sonicated. After centrifugation at $11,000 \mathrm{rpm}$ for $1 \mathrm{~h}$ at $4{ }^{\circ} \mathrm{C}$, ASC was present in inclusion bodies (pellet). Pellets were then resuspended in $100 \mathrm{ml}$ of denaturing Buffer $\mathrm{B}\left(50 \mathrm{mM} \mathrm{NaH}{ }_{2} \mathrm{PO}_{4}, 300 \mathrm{mM} \mathrm{NaCl}, 8 \mathrm{M}\right.$ urea, $\mathrm{pH}$ 8.0) and resonicated. Under these conditions, the His tagged-ASC protein was solubilized and purified using TALON resin (Qiagen). Re-folding was performed in the TALON resin at a gradient of decreasing urea concentrations, and the protein was finally eluted in $15 \mathrm{ml}$ of Buffer $\mathrm{C}\left(50 \mathrm{mM} \mathrm{NaH} \mathrm{PO}_{4} \mathrm{pH} 8.0\right.$, $300 \mathrm{mM} \mathrm{NaCl}, 500 \mathrm{mM}$ imidazole). Desalting was performed in a PD-10 desalting column, and the protein was finally concentrated in a $10 \mathrm{~K}$ Amicon concentrator unit (Merck Millipore).

\section{Primary screening: ASC-mediated pro-Casp-1 reconstitution in vitro assay}

Recombinant ASC $(300 \mathrm{nM})$ was pre-incubated with the indicated concentration of compounds for $20 \mathrm{~min}$ at room temperature (RT) in Assay Buffer (20 mM HEPES pH 7.5, $10 \mathrm{mM} \mathrm{KCl}, 1.5 \mathrm{mM} \mathrm{MgCl}, 1 \mathrm{mM}$ EDTA, $1 \mathrm{mM}$ EGTA, $1 \mathrm{mM}$ DTT). Then pro-Casp-1 $(50 \mathrm{nM})$ was added and incubated for another $10 \mathrm{~min}$. Caspase-1 activation was measured using the Ac-WEHD-AFC fluorogenic substrate $(80 \mu \mathrm{M}$; PeptaNova) by continuously monitoring the release of AFC in a Wallac Victor workstation. The MyriaScreen Diversity Library ${ }^{\circledR}$ (Sigma-Aldrich) comprises 10,000 drugs dissolved in dimethyl sulfoxide (DMSO), selected to maximize the chemical diversity while maintaining drug-like properties. The compounds were 
A

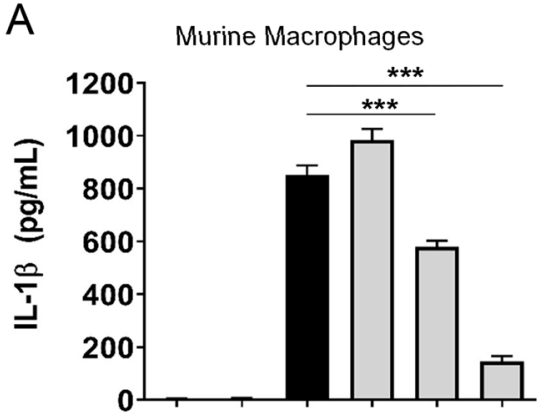

C

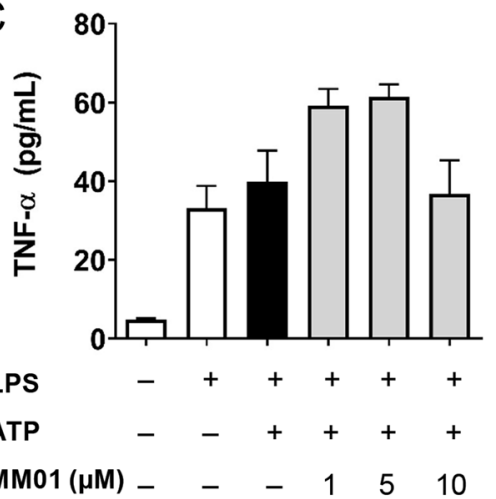

E

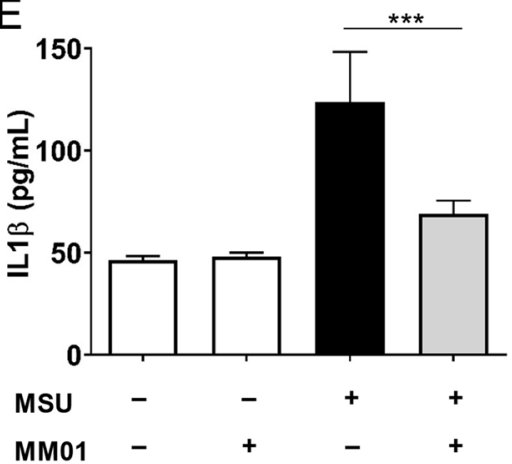

B

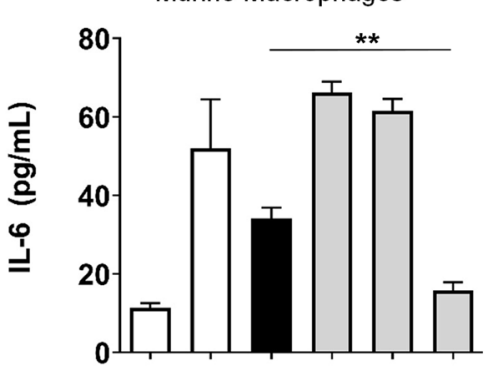

D

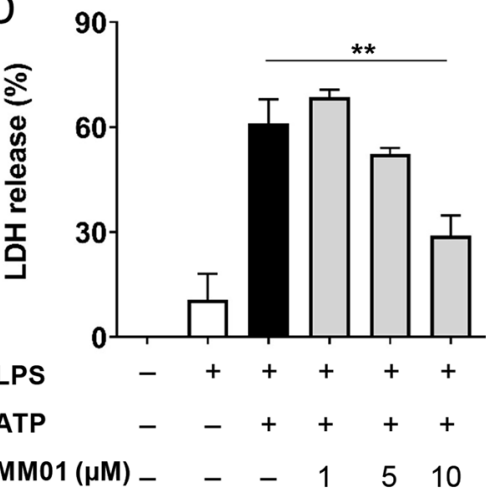

F

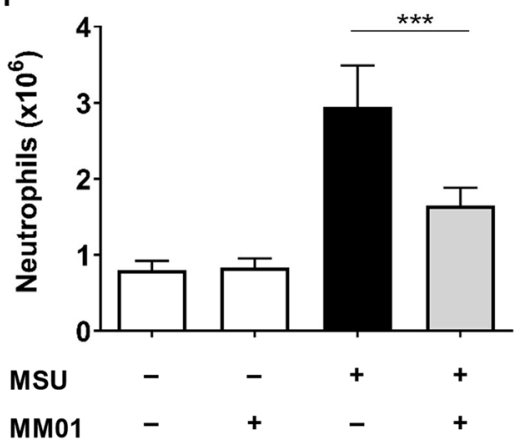

Fig. 5 MM01 inhibits ASC-dependent inflammasome activity in stimulated murine peritoneal macrophages and in MSU-induced peritonitis mouse model. IL-1 $\beta$ (A), IL-6 (B), and TNF- $\alpha$ (D) secretion was evaluated by ELISA upon activation of the NLRP3 inflammasome with LPS $(100 \mathrm{ng} / \mathrm{ml})$ and ATP $(2.5 \mathrm{mM})+/-\mathrm{MM} 01$ at 1,5 , or $10 \mu \mathrm{M}$ in isolated peritoneal macrophages. C Percentage of LDH release under the above-described conditions. In all cases, asterisks represent significant differences to the stimulated control (LPS/ATP), as determined by a one-way ANOVA with Tukey's multiple comparisons test ${ }^{* *} p<0.05 ;{ }^{* *} p<0.001$. All data expressed as mean \pm SD of three experiments. E IL-1 $\beta$ detection by ELISA in the peritoneal cavity of C57BL/6 mice injected with MSU crystals with or without MM01 (10 mg/kg) treatment. F Neutrophil numbers in the peritoneal cavity of C57BL/6 mice treated as in the above-described conditions. Data are representative of two independent experiments (mean \pm SD of $n=12$ ). Asterisks represent significant differences to the stimulated control as determined by a oneway ANOVA with Tukey's multiple comparisons test ${ }^{* *} p<0.001$.

evaluated at $20 \mu \mathrm{M}$ with $1 \%$ of DMSO in dual-mixes for cost-effectivity reasons. Deconvolution took place by testing individually the compounds that made up each of the positive mixtures. Data are reported as the percentage of inhibition compared with the absence of the compound.

\section{Secondary screening: caspase-1 in vitro activation assay}

Caspases have a high processivity rate. Base on this, active caspase-1 can be purified as indicated above for pro-Casp-1 recombinant protein but increasing infection time to $24 \mathrm{~h}$. Once protein is active, activity can be monitored with a fluorogenic substrate. Recombinant caspase-1 (50 nM) was incubated with the indicated concentrations of compounds for $20 \mathrm{~min}$ at RT in Assay Buffer. Then Ac-WEHD-AFC fluorogenic substrate $(80 \mu \mathrm{M})$ was added, and the activity was monitored in a Wallac Victor workstation. Compounds able to inhibit Casp-1 activity were discarded. Data are reported as the percentage of inhibition compared with the absence of the inhibitor.

\section{ASC filament formation in vitro}

Recombinant human ASC protein was eluted from PD-10 column in an acid $\mathrm{pH}$ buffer to avoid precipitation. Then we induce filament formation achieving neutral $\mathrm{pH}$ by adding $3 \mathrm{M}$ Tris buffer ( $\mathrm{pH} 8.0$ ) 1:5 ratio ( $\mathrm{vol} / \mathrm{vol})$. ASC samples $(12 \mu \mathrm{M})$ were incubated overnight at room temperature. A drop of $10 \mu \mathrm{l}$ sample were placed on clean Parafilm and a mesh copper pure carbon coated grid were floated on top for $10 \mathrm{~min}$. Then, the grids were transferred and contrasted with 1\% uranyl acetate for 5 min. Excess fluid was removed and allowed to dry before examination with a transmission electron microscope FEI Tecnai G2 Spirit (ThermoFisher Scientific Company, OR, USA). All images were acquired using the Radius software with a digital camera Xarosa (EMSIS GmbH, Münster, Germany).

\section{Cell cultures}

HEK293 cells (CRL-1573; ATCC) were grown in Dulbecco's Modified Eagle Medium (DMEM)-high glucose medium supplemented with $10 \%$ fetal 
bovine serum (FBS). THP-1 (TIB-202; ATCC) cells were grown as supplier recommended. THP1-ASC-GFP were also cultured as supplier recommended (thp-ascgfp; InvivoGen). All cultures were maintained at $37^{\circ} \mathrm{C}$ in a $5 \% \mathrm{CO}_{2}$ atmosphere and were checked for mycoplasma contamination every 6 months.

\section{ASC speck assay}

THP1-ASC-GFP cells were seeded at $1 \times 10^{6}$ cells $/ \mathrm{ml}$ the day before use in experiments on $35 \mathrm{~mm}$ glass-bottom culture dishes. The following day, the culture medium was replaced with fresh medium containing MM01 $(10 \mu \mathrm{M})$ or vehicle for $30 \mathrm{~min}$. Cells were then primed with $100 \mathrm{ng} / \mathrm{ml}$ LPS for $3 \mathrm{~h}$ and stimulated with $10 \mu \mathrm{M}$ nigericin for $30 \mathrm{~min}$. For confocal analysis, samples were fixed in $4 \%$ paraformaldehyde for $10 \mathrm{~min}$ at RT, washed several times, and prepared in mounting medium plus DAPI. Image acquisition used a Leica SP8 confocal microscope, and image processing was performed using the FiJi software. For cytometry analysis, samples were detached and analyzed in a CytoFlex Flow Cytometer (Beckman Coulter).

\section{ASC oligomerization assays}

PMA-differentiated THP-1 cells were seeded at $1 \times 10^{6}$ cells $/ \mathrm{ml}$ in 6-well plates. Cells were treated with MM01 $(10 \mu \mathrm{M})$ for $30 \mathrm{~min}$, primed with $100 \mathrm{ng} / \mathrm{ml}$ LPS for $3 \mathrm{~h}$, and then stimulated with $10 \mu \mathrm{M}$ nigericin for $30 \mathrm{~min}$. The supernatants were removed, cells were rinsed in ice-cold PBS and then lysed in NP-40 buffer (20 mM HEPES-KOH pH 7.5, $150 \mathrm{mM} \mathrm{KCL}, 1 \% \mathrm{NP}-40$ plus protease inhibitors). Lysates were centrifuged at $330 \times g$ for $10 \mathrm{~min}$ at $4{ }^{\circ} \mathrm{C}$. The pellets were washed and resuspended in PBS plus $2 \mathrm{mM}$ disuccinimidyl suberate and incubated at RT for $30 \mathrm{~min}$ with rotation. Samples were then centrifuged at $330 \times g$ for $10 \mathrm{~min}$ at $4^{\circ} \mathrm{C}$. The supernatant was removed, and the crosslinked pellets were then resuspended in sample buffer, boiled, and analyzed by immunoblotting.

HEK293 cells were seeded at $3.0 \times 10^{5}$ cells $/ \mathrm{ml}$ in 6-well plates and transfected with ASC-YFP construct [22] according to standard procedure for Lipofectamine 2000 reagent (Invitrogen). Then cells were treated with MM01 $(10 \mu \mathrm{M})$ for $6 \mathrm{~h}$ and oligomers purified as described above. Bafilomycin A1 (InvivoGen) is a selective inhibitor of vacuolar-type H + ATPase (V-ATPase) that inhibits autophagosome and lysosome fusion. Bafilomycin (200 nM) treatment for $2 \mathrm{~h}$ was used as an inhibitor of protein degradation.

\section{ASC mutant analysis}

ASC mutants in the active site predicted by MM01 molecular docking were generated by using the QuikChange Site-Directed Mutagenesis Kit (Agilent Technologies) in the ASC-YFP plasmid. These mutations include $\mathrm{H} 118 \mathrm{~A}$, F163A, W169A, K174A, L192A, R119A, the double mutant L177A-L178A, and the binding site mutant (BS) containing all mentioned mutations. All molecular biology techniques were performed according to the standard procedures.

To assess the effect of ASC mutants on speck formation, HEK293 cells were seeded at a concentration of $4.0 \times 10^{5}$ cells $/ \mathrm{ml}$ in 12 -well plates. The next day, Lipofectamine ${ }^{\mathrm{TM}} 2000$ (Invitrogen) was used according to the manufacturer's instructions to transfect the cells with the ASC-YFP plasmid. After 24 h, HEK293 cells were harvested to analyze the speck formation by flow cytometry and confocal microscopy as well as the oligomerization formation by chemical crosslinking. In addition, whole-cell extracts were obtained to check the presence of the fusion protein in every sample.

\section{Docking calculations with GOLD 5.2}

The crystal structure of human ASC (PDB ID: 2KN6) was downloaded from the protein data bank2 and subjected to docking calculations using the GOLD 5.2 software (CCDC, Cambridge, UK). The internal energy of the compounds was first minimized using the MM2 protocol and submitted to GOLD in SDF format. Docking experiments were performed three times using the default docking settings and ChemScore as the scoring function. A total of 30 genetic algorithms (GA) runs were set for each compound. To accelerate the calculations, the program was allowed to stop the GA runs when the top three solutions were within $1.5 \AA$ root mean square deviation. Intermolecular interactions were described using Discovery Studio 4.0 (Accelrys Inc., San Diego, CA, USA).

\section{Cellular assay for inflammasome activation}

The molecules were evaluated in THP-1 or PMA-differentiated THP-1 cells $(50 \mathrm{nM}$ PMA for $24 \mathrm{~h})$ stimulated with LPS $(100 \mathrm{ng} / \mathrm{ml})$ and MDP $(50 \mu \mathrm{g} / \mathrm{ml})$ or with LPS plus adenosine triphosphate (2.5 mM ATP) to stimulate ASCdependent inflammasomes NLRP1 and NLRP3, respectively. Briefly, $1 \times 10^{6}$ cells were seeded in 6-well plates in $1 \mathrm{ml}$ RPMI media that contained $1 \%$ FBS. Cells were either mock-treated or primed with compounds at the indicated concentrations for $30 \mathrm{~min}$, followed by treatment with LPS (Sigma) and MDP (Sigma) or ATP (Sigma) for $6 \mathrm{~h}$ at $37^{\circ} \mathrm{C}$. In the case of nigericin stimulation, cells were primed with LPS for $3 \mathrm{~h}$, and then $10 \mu \mathrm{M}$ nigericin (Sigma) was added for $30 \mathrm{~min}$. Treatment with $10 \mu \mathrm{M}$ MM01 was optimal in cellular assays due to the intrinsic characteristics of the compound in the cellular environment. Supernatants were harvested and clarified by centrifugation at $1500 \mathrm{rpm}$ at RT, and cytokine analysis was then performed. To induce NLRC4 inflammasome, cells were pretreated with the compounds for $30 \mathrm{~min}$, and then cells were stimulated for $3 \mathrm{~h}$ with Salmonella Typhimurium ( $\mathrm{MOI}$ 5). To induce AIM2 inflammasomes, cells were pretreated in the same conditions as before and transfected with poly(dA:dT) $(0.5 \mu \mathrm{g} / \mathrm{ml}$; Sigma) overnight using Lipofectamine 2000 (Invitrogen).

Both IL-1 $\beta$ secretion and the release of IL-18 were monitored by enzyme-linked immunosorbent assay (ELISA; 557953 BD OptEIA ${ }^{\mathrm{TM}}$ and BMS267-2 from eBioscience) following the manufacturer's instructions. Cell viability was analyzed in parallel by evaluating the release of LDH according to a commercial kit (CytoTox-ONE ${ }^{\mathrm{TM}}$ Homogeneous Membrane Integrity Assay G7890; Promega). The release of LDH was calculated using the formula: the release of LDH $(\%)=100 \times$ (Abs490 treated - Abs490 untreated cells)/Abs490 untreated cells lysed with Triton 9\% (maximum release of $L D H)$.

\section{Murine macrophage and PBMC assays}

Human PBMCs were purchased from Lonza (CC-2702). PBMCs were plated in 24-well plates at 5,000,000 cells/well in serum-free RPMI medium. Cells were primed for $3 \mathrm{~h}$ with $200 \mathrm{ng} / \mathrm{ml}$ pure LPS followed by $30 \mathrm{~min}$ with $10 \mu \mathrm{M} \mathrm{MM01}$ and $1 \mathrm{~h}$ with $10 \mu \mathrm{M}$ nigericin. Supernatants were harvested and clarified by centrifugation at $1500 \mathrm{rpm}$ at RT, and cytokine and caspase-1 activation analysis was performed as previously described.

Mice (BALB/c) were euthanized, and $5 \mathrm{ml}$ of cold medium was injected (20 gauge needle) into the peritoneal region. The aspirated fluid from the peritoneum was centrifuged for $10 \mathrm{~min}$ at $400 \times g$ and the cell pellet was dissolved in cold DMEM/F12-10 (1 ml). Isolation of macrophages was performed according to a previously described protocol [56].

Macrophages were plated in 24-well plates at 400,000 cells/well overnight. Cells were primed for $3 \mathrm{~h}$ with $200 \mathrm{ng} / \mathrm{ml}$ pure LPS (Alexis Biochemicals Corporation) in serum-free IMDM media followed by $30 \mathrm{~min}$ with the compounds and $5 \mathrm{mM}$ ATP. Supernatants were harvested and clarified by centrifugation at $1500 \mathrm{rpm}$ at RT, and cytokine and caspase-1 activation analysis was performed as previously described for the THP-1 cell line.

\section{Immunoblotting}

The supernatants of the treated cells were precipitated by the chloroform-methanol method, as previously described [34]. Pellets were obtained by lysing cells in $25 \mathrm{mM}$ of Tris- $\mathrm{HCl} \mathrm{pH} \mathrm{7.4,1} \mathrm{mM} \mathrm{EDTA,} 1 \mathrm{mM}$ EGTA, and $1 \%$ sodium dodecyl sulfate (SDS), plus protease and phosphatase inhibitors. The protein concentration was determined by the BCA protein assay. Samples were separated in a $14 \%$ SDSpolyacrylamide gel electrophoresis gel, transferred to a nitrocellulose membrane, and blocked with 5\% skimmed milk for $1 \mathrm{~h}$. Then the membrane was incubated overnight with primary antibodies: a-casp1 (1:1000; Cell Signaling 2225), $a-I L-1 \beta$ (1:1000; Acris R1130P), a-NLRP1 (1:1000; ALX-210-904-R100), a-NLRP3 (1:1000; Cell Signaling 15101), a-ASC (1:1000; Santa Cruz Biotechnology sc-22514-R), a-gapdh (1:1000; Cell Signaling 2118), and a-tubulin (1:3000; Abcam ab6160) at $4{ }^{\circ} \mathrm{C}$. Membranes were washed and probed with the appropriate secondary antibody conjugated with peroxidase for enhanced chemiluminescence detection (Amersham Pharmacia Biotech).

\section{MSU-induced peritonitis mouse model}

In vivo model was performed as described in Bioprotocols [41] using an approach approved by the Institutional Animal Ethical Committee (CEEA 2018_28). C57BL/6 mice (9 weeks) were injected intraperitoneally with $10 \mathrm{mg} / \mathrm{kg} \mathrm{MM01}$ or vehicle (PBS + 0.1\% DMSO) $30 \mathrm{~min}$ before intraperitoneal injection of MSU ( $2 \mathrm{mg} \mathrm{MSU}$ crystals in $200 \mu \mathrm{l}$ sterile PBS). After $6 \mathrm{~h}$, mice were sacrificed, and peritoneal lavage with $5 \mathrm{ml}$ of PBS was performed. Cytokine IL-1 $\beta$ (DY401 R\&D Systems), TNF-a (555268 BD 
OptEIA ${ }^{\mathrm{TM}}$ Set), and IL-6 (555240 BD OptEIA ${ }^{\mathrm{TM}}$ Set) secretion was determined by ELISA, and neutrophil content measured by flow cytometry in ACVLAB Laboratorio Valencia. A total of $N=12$ animals per condition was used. Groups were distributed randomly without any specific methodology. No blinding was done. Experiment was done two times, 50\% males and 50\% females. Sample size was calculated using the GRANMO Software (https:// www.imim.es/ofertadeserveis/software-public/granmo).

\section{Statistical analysis}

Data are expressed as mean $\pm S D$ and the number of biological replicates is indicated in each figure legend. Data were analyzed with the GraphPad Prism software. Statistical significance $\left({ }^{* *} p<0.05 ;{ }^{* * *} p<0.01\right)$ was assessed by Student's $t$ test when used to compare two groups. When comparing more than two groups, a one-way analysis of variance test with Tukey's multiple post test comparisons was used to identify differences.

\section{DATA AVAILABILITY}

All relevant data are within the manuscript and its Supplementary Information files.

\section{REFERENCES}

1. Martinon F, Burns K, Tschopp J. The inflammasome: a molecular platform triggering activation of inflammatory caspases and processing of prolL-beta. Mol Cell. 2002;10:417-26.

2. Medzhitov R. Origin and physiological roles of inflammation. Nature. 2008;454:428-35.

3. Lamkanfi M, Dixit VM. Inflammasomes and their roles in health and disease. Annu Rev Cell Dev Biol. 2012;28:137-61.

4. Friker LL, Scheiblich H, Hochheiser IV, Brinkschulte R, Riedel D, Latz E, et al. betaAmyloid clustering around ASC fibrils boosts its toxicity in microglia. Cell Rep. 2020;30:3743.e6-54.e6.

5. Olsen I, Singhrao SK. Inflammasome involvement in Alzheimer's disease. J Alzheimers Dis. 2016;54:45-53.

6. Masters SL, Dunne A, Subramanian SL, Hull RL, Tannahill GM, Sharp FA, et al. Activation of the NLRP3 inflammasome by islet amyloid polypeptide provides a mechanism for enhanced IL-1beta in type 2 diabetes. Nat Immunol. 2010;11:897-904.

7. Ghiringhelli F, Apetoh L, Tesniere A, Aymeric L, Ma Y, Ortiz C, et al. Activation of the NLRP3 inflammasome in dendritic cells induces IL-1beta-dependent adaptive immunity against tumors. Nat Med. 2009;15:1170-8.

8. Guo B, Fu S, Zhang J, Liu B, Li Z. Targeting inflammasome/IL-1 pathways for cancer immunotherapy. Sci Rep. 2016;6:36107.

9. Heymann MC, Rosen-Wolff A. Contribution of the inflammasomes to autoinflammatory diseases and recent mouse models as research tools. Clin Immunol. 2013;147:175-84.

10. Masters SL. Specific inflammasomes in complex diseases. Clin Immunol. 2013;147:223-8.

11. Moll M, Kuemmerle-Deschner JB. Inflammasome and cytokine blocking strategies in autoinflammatory disorders. Clin Immunol. 2013;147:242-75.

12. Chang YS, Ko BH, Ju JC, Chang HH, Huang SH, Lin CW. SARS unique domain (SUD) of severe acute respiratory syndrome coronavirus induces NLRP3 inflammasomedependent CXCL10-mediated pulmonary inflammation. Int $\mathrm{J}$ Mol Sci. 2020;21:3179.

13. Bianchi ME. DAMPs, PAMPs and alarmins: all we need to know about danger. J Leukoc Biol. 2007;81:1-5.

14. de Alba E. Structure, interactions and self-assembly of ASC-dependent inflammasomes. Arch Biochem Biophys. 2019;670:15-31.

15. Dick MS, Sborgi L, Ruhl S, Hiller S, Broz P. ASC filament formation serves as a signal amplification mechanism for inflammasomes. Nat Commun. 2016;7:11929.

16. Li Y, Fu TM, Lu A, Witt K, Ruan J, Shen C, et al. Cryo-EM structures of ASC and NLRC4 CARD filaments reveal a unified mechanism of nucleation and activation of caspase-1. Proc Natl Acad Sci USA. 2018;115:10845-52.

17. Proell M, Gerlic M, Mace PD, Reed JC, Riedl SJ. The CARD plays a critical role in ASC foci formation and inflammasome signalling. Biochem J. 2013;449:613-21.

18. Chen G, Shaw MH, Kim YG, Nunez G. NOD-like receptors: role in innate immunity and inflammatory disease. Annu Rev Pathol. 2009;4:365-98.

19. Kayagaki N, Stowe IB, Lee BL, O'Rourke K, Anderson K, Warming S, et al. Caspase11 cleaves gasdermin $D$ for non-canonical inflammasome signalling. Nature. 2015;526:666-71.

20. Liu X, Zhang Z, Ruan J, Pan Y, Magupalli VG, Wu H, et al. Inflammasome-activated gasdermin $D$ causes pyroptosis by forming membrane pores. Nature. 2016;535:153-8.

21. Shi J, Zhao $Y$, Wang $K$, Shi $X$, Wang $Y$, Huang $H$, et al. Cleavage of GSDMD by inflammatory caspases determines pyroptotic cell death. Nature. 2015;526:660-5.
22. Baroja-Mazo A, Martin-Sanchez F, Gomez Al, Martinez CM, Amores-Iniesta J, Compan V, et al. The NLRP3 inflammasome is released as a particulate danger signal that amplifies the inflammatory response. Nat Immunol. 2014;15:738-48.

23. He XF, Zeng YX, Li G, Feng YK, Wu C, Liang FY, et al. Extracellular ASC exacerbated the recurrent ischemic stroke in an NLRP3-dependent manner. J Cereb Blood Flow Metab. 2020;40:1048-60.

24. Xu Z, Zhou Y, Liu M, Ma H, Sun L, Zahid A, et al. Homotypic CARD-CARD interaction is critical for the activation of NLRP1 inflammasome. Cell Death Dis. 2021;12:57.

25. Saresella M, Piancone F, Marventano I, Zoppis M, Hernis A, Zanette M, et al. Multiple inflammasome complexes are activated in autistic spectrum disorders. Brain Behav Immun. 2016;57:125-33.

26. Freeman L, Guo H, David CN, Brickey WJ, Jha S, Ting JP. NLR members NLRC4 and NLRP3 mediate sterile inflammasome activation in microglia and astrocytes. J Exp Med. 2017;214:1351-70.

27. Ravi Kumar S, Paudel S, Ghimire L, Bergeron S, Cai S, Zemans RL, et al. Emerging roles of inflammasomes in acute pneumonia. Am J Respir Crit Care Med. 2018;197:160-71.

28. Yang $Y$, Wang $H$, Kouadir $M$, Song $H$, Shi F. Recent advances in the mechanisms of NLRP3 inflammasome activation and its inhibitors. Cell Death Dis. 2019;10:128.

29. Wannamaker W, Davies R, Namchuk M, Pollard J, Ford P, Ku G, et al. (S)-1-((S)-2\{[1-(4-amino-3-chloro-phenyl)-methanoyl]-amino\}-3,3-dimethyl-butanoy I)-pyrrolidine-2-carboxylic acid ((2R,3S)-2-ethoxy-5-oxo-tetrahydro-furan-3-yl)-amide (VX765), an orally available selective interleukin (IL)-converting enzyme/caspase-1 inhibitor, exhibits potent anti-inflammatory activities by inhibiting the release of IL-1beta and IL-18. J Pharmacol Exp Ther. 2007;321:509-16.

30. Dinarello CA, Simon A, van der Meer JW. Treating inflammation by blocking interleukin-1 in a broad spectrum of diseases. Nat Rev Drug Discov. 2012;11:633-52.

31. Portal OC. 2012. http://www.organic-chemistry.org/prog/peo/. Accessed 22 May 2020.

32. Hoss F, Rolfes V, Davanso MR, Braga TT, Franklin BS. Detection of ASC speck formation by flow cytometry and chemical cross-linking. Methods Mol Biol. 2018;1714:149-65

33. Le Gall JY, Khoi TD, Glaise D, Le treut A, Brissot P, Guillouzo A. Lysosomal enzyme activities during ageing of adult human liver cell lines. Mech Ageing Dev. 1979;11:287-93.

34. De Nardo CM, Latz E. The inflammasome. Berlin: Springer; 2013.

35. Jones G, Willett $P$, Glen RC, Leach AR, Taylor R. Development and validation of a genetic algorithm for flexible docking. J Mol Biol. 1997;267:727-48.

36. Schneider KS, Gross CJ, Dreier RF, Saller BS, Mishra R, Gorka O, et al. The inflammasome drives GSDMD-independent secondary pyroptosis and IL-1 release in the absence of caspase-1 protease activity. Cell Rep. 2017;21:3846-59.

37. Mariathasan S, Weiss DS, Newton $K$, McBride J, O'Rourke $K$, Roose-Girma M, et al. Cryopyrin activates the inflammasome in response to toxins and ATP. Nature. 2006;440:228-32.

38. Gregory SM, Davis BK, West JA, Taxman DJ, Matsuzawa S, Reed JC, et al. Discovery of a viral NLR homolog that inhibits the inflammasome. Science. 2011;331:330-4.

39. Man SM, Tourlomousis P, Hopkins L, Monie TP, Fitzgerald KA, Bryant CE. Salmonella infection induces recruitment of caspase- 8 to the inflammasome to modulate IL-1beta production. J Immunol. 2013;191:5239-46.

40. Cahill CM, Rogers JT. Interleukin (IL) 1 beta induction of IL-6 is mediated by a novel phosphatidylinositol 3-kinase-dependent AKT/lkappaB kinase alpha pathway targeting activator protein-1. J Biol Chem. 2008;283:25900-12.

41. Spalinger MRaS M. Mono sodium urate crystal-induced peritonitis for in vivo assessment of inflammasome activation. Bio Protoc. 2018;8:e2754.

42. Martinon F, Petrilli V, Mayor A, Tardivel A, Tschopp J. Gout-associated uric acid crystals activate the NALP3 inflammasome. Nature. 2006;440:237-41.

43. Spalinger MR, Kasper S, Gottier C, Lang S, Atrott K, Vavricka SR, et al. NLRP3 tyrosine phosphorylation is controlled by protein tyrosine phosphatase PTPN22. J Clin Investig. 2016;126:1783-1800.

44. Nowarski R, Jackson R, Gagliani N, de Zoete MR, Palm NW, Bailis W, et al. Epithelial IL-18 equilibrium controls barrier function in colitis. Cell. 2015;163:1444-56.

45. Schmidt FI, Lu A, Chen JW, Ruan J, Tang C, Wu H, et al. A single domain antibody fragment that recognizes the adaptor ASC defines the role of ASC domains in inflammasome assembly. J Exp Med. 2016;213:771-90.

46. Desu HL, Plastini M, Illiano P, Bramlett HM, Dietrich WD, de Rivero Vaccari JP, et al. IC100: a novel anti-ASC monoclonal antibody improves functional outcomes in an animal model of multiple sclerosis. J Neuroinflammation. 2020;17:143.

47. Cheong M, Gartlan KH, Lee JS, Tey SK, Zhang P, Kuns RD, et al. ASC modulates CTL cytotoxicity and transplant outcome independent of the inflammasome. Cancer Immunol Res. 2020;8:1085-98.

48. Chen GY, Liu M, Wang F, Bertin J, Nunez G. A functional role for Nlrp6 in intestinal inflammation and tumorigenesis. J Immunol. 2011;186:7187-94.

49. Hu B, Elinav E, Huber S, Booth CJ, Strowig T, Jin C, et al. Inflammation-induced tumorigenesis in the colon is regulated by caspase- 1 and NLRC4. Proc Natl Acad Sci USA. 2010;107:21635-40. 
50. Zaki MH, Lamkanfi M, Kanneganti TD. The Nlrp3 inflammasome: contributions to intestinal homeostasis. Trends Immunol. 2011;32:171-9.

51. Henao-Mejia J, Elinav E, Jin C, Hao L, Mehal WZ, Strowig T, et al. Inflammasomemediated dysbiosis regulates progression of NAFLD and obesity. Nature. 2012;482:179-85.

52. Coll RC, Robertson AA, Chae JJ, Higgins SC, Munoz-Planillo R, Inserra MC, et al. A small-molecule inhibitor of the NLRP3 inflammasome for the treatment of inflammatory diseases. Nat Med. 2015;21:248-55.

53. Jiang $\mathrm{H}, \mathrm{He} \mathrm{H}$, Chen $\mathrm{Y}$, Huang W, Cheng J, Ye J, et al. Identification of a selective and direct NLRP3 inhibitor to treat inflammatory disorders. J Exp Med. 2017:214:3219-38.

54. Gram AM, Frenkel J, Ressing ME. Inflammasomes and viruses: cellular defence versus viral offence. J Gen Virol. 2012;93:2063-75.

55. Toldo S, Bussani R, Nuzzi V, Bonaventura A, Mauro AG, Cannata A, et al. Inflammasome formation in the lungs of patients with fatal COVID-19. Inflamm Res. 2021;70:7-10.

56. Zhang X, Goncalves R, Mosser DM. The isolation and characterization of murine macrophages. Curr Protoc Immunol. 2008;Chapter 14:Unit 14.11.

\section{ACKNOWLEDGEMENTS}

We thank Alicia García for technical support.

\section{AUTHOR CONTRIBUTIONS}

Conceptualization: MS, MO, and PP; methodology: PMS-T, GG-L, MS, MA, and CD; data analysis: GGL, PMS-T, and MS; writing: MS and MO; supervision: JP, IM, MJV, MS, and MO. All authors read and approved the final manuscript.

\section{FUNDING}

This work was supported by grants from the Spanish Ministry of Economy and Competitiveness (Grants SAF2017-84689-R and SAF2017-88276-R to PP), the European Research Council (ERC-2013-CoG grant 614578 to PP), and the Generalitat Valenciana (PROMETEO/2019/065). GG-L was funded by a predoctoral fellowship from JAE-pre CSIC from the Spanish government (JaePre021). PMS-T was funded by a predoctoral fellowship from CIPF. CIPF is a specialized screening site within the ERIC EU-OpenScreen. Part of the equipment employed in this work has been funded by Generalitat Valenciana and co-financed with FEDER funds (PO FEDER of Comunitat Valenciana 2014-2020)
ETHICS APPROVAL AND CONSENT TO PARTICIPATE

All animal experiments were approved by the Institutional Animal Ethical Committee (No. 2018_28).

\section{COMPETING INTERESTS}

Compound MM01 is under patent procedure: patent applicant CENTRO DE INVESTIGACIÓN PRINCIPE FELIPE, name of inventor(s), Mar Orzáez, Mónica Sancho, Paula M Soriano-Teruel, Guillermo García-Laínez; application number, 20382237.41109; status of application, registered.

\section{ADDITIONAL INFORMATION}

Supplementary information The online version contains supplementary material available at https://doi.org/10.1038/s41419-021-04420-1.

Correspondence and requests for materials should be addressed to Mónica Sancho or Mar Orzáez.

Reprints and permission information is available at http://www.nature.com/ reprints

Publisher's note Springer Nature remains neutral with regard to jurisdictional claims in published maps and institutional affiliations.

(C) Open Access This article is licensed under a Creative Commons Attribution 4.0 International License, which permits use, sharing, adaptation, distribution and reproduction in any medium or format, as long as you give appropriate credit to the original author(s) and the source, provide a link to the Creative Commons license, and indicate if changes were made. The images or other third party material in this article are included in the article's Creative Commons license, unless indicated otherwise in a credit line to the material. If material is not included in the article's Creative Commons license and your intended use is not permitted by statutory regulation or exceeds the permitted use, you will need to obtain permission directly from the copyright holder. To view a copy of this license, visit http://creativecommons. org/licenses/by/4.0/.

(c) The Author(s) 2021 